Article

\title{
Rh(III)-catalyzed coupling of nitrones with alkynes for the synthesis of indolines
}

\author{
Lingheng Kong, Fang Xie, Songjie Yu, Zisong Qi, Xingwei Li* \\ Dalian Institute of Chemical Physics, Chinese Academy of Sciences, Dalian 116023, Liaoning, China
}

\section{A R T I C L E I N F O}

Article history:

Received 22 April 2015

Accepted 30 April 2015

Published 20 July 2015

Keywords:

Rhodium(III)

Carbon-hydrogen activation

Oxygen-atom transfer

Nitrogen-aryl nitrones

\section{A B S T R A C T}

Rh-catalyzed redox-neutral coupling between $N$-aryl nitrones and alkynes has been achieved under relatively mild conditions. The reaction proceeded via $\mathrm{C}-\mathrm{H}$ activation at the $\mathrm{N}$-aryl ring with subsequent $\mathrm{O}$-atom transfer, affording trisubstituted indolines in good chemoselectivity and moderate to good diasteroselectivity.

(C) 2015, Dalian Institute of Chemical Physics, Chinese Academy of Sciences. Published by Elsevier B.V. All rights reserved.

\section{Introduction}

Metal-catalyzed $\mathrm{C}-\mathrm{H}$ activation of arenes has received increasing attention in the past several decades and this strategy has allowed the development of a plethora of new synthetic methods to access complex structures [1-8]. This strategy is appealing in that no prior functionalization of arenes is necessary. In most cases, the $\mathrm{C}-\mathrm{H}$ activation of less intrinsically active arenes requires the installation of a directing group typically at the ortho position [9-14]. The $\mathrm{C}-\mathrm{H}$ bond is cleaved with concurrent formation of a metalacycle, which provides a driving force to enhance both reactivity and selectivity. Despite the progress, the presence of a pendent directing group in the coupled product is often undesirable. To address this limitation, a number of coupling systems with functionalizable or transferable DGs in arene substrates have been developed [15]. However, challenges remain and it is necessary to develop more efficient and selective $\mathrm{C}-\mathrm{H}$ activation systems by taking advantage of multi-task DGs.

In this context, stable $\mathrm{Cp} \mathrm{p}^{*} \mathrm{Rh}(\mathrm{III})$ complexes have stood out as efficient catalysts for the selective $\mathrm{C}-\mathrm{H}$ functionalization of a large scope of arenes [16-19], and this area has experienced tremendous progress. In particular, Fagnou and Glorius pioneered in $\mathrm{C}-\mathrm{H}$ activation of arenes assisted by an oxidizing $\mathrm{N}-\mathrm{O}$ bond as a DG under redox-neutral conditions [20-24]. These systems overcame limited roles of DGs in general because they participated in subsequent reactions as internal oxidants, especially leading to efficient synthesis of heterocycles under mild conditions when alkenes and alkynes were used as the coupling partner [25-36]. In addition, these reactions are redox-economic since no external oxidant is necessary. Inspired by this strategy, the oxidizing DGs have been extended to other oxidizing bonds such as N-N [37-44], N-S [45], and C-N [46]. On the other hand, the cleavage of these oxidizing groups almost always generates a small molecule such as water, alcohol, acid, or amine as a co-product [25-46], which limited the at-

\footnotetext{
* Corresponding author. Tel: +86-411-84379089; E-mail: xwli@dicp.ac.cn This work was supported by the Dalian Institute of Chemical Physics, Chinese Academy of Sciences, and the National Natural Science Foundation of China $(21272231,21472186,21372266)$.
} 
om-economy of the coupling system.

We recently reported the $\mathrm{Rh}(\mathrm{III})$-catalyzed redox-neutral coupling between quinolone $N$-oxides and alkynes, leading to the synthesis of diaryl-substituted acetophenones [47,48]. In this reaction, the $\mathrm{N}-\mathrm{O}$ bond acted as an oxidizing DG to realize $\mathrm{C}-\mathrm{H}$ activation at the 8-position. Significantly, the oxygen was retained via an intramolecular OAT process with $100 \%$ atom-economy. So far this $\mathrm{C}-\mathrm{H}$ activation-OAT system has been achieved for only one arene system. It is necessary to extend this strategy to other related arenes for the preparation of synthetically useful complex products. Arenes such as benzaldehyde-derived nitrones are structurally related to quinolone $N$-oxides, and our previous studies revealed that $\mathrm{N}$-tert-butyl- $\alpha$-arylnitrones coupled with alkynes under $\mathrm{Rh}$ catalysis to afford indenones [49]. However, no OAT to alkyne was involved although the polar $\mathrm{N}-\mathrm{O}$ bond proved to be a viable DG to effect endo $\mathrm{C}-\mathrm{H}$ activation. On the other hand, Wan and coworkers reported in one example the exo-type $\mathrm{C}-\mathrm{H}$ activation of nitrone in the coupling with diphenylacetylene, affording an indole [50-53]. We reasoned that $N$-aryl nitrone may still undergo $\mathrm{C}-\mathrm{H}$ activation-OTA coupling with alkynes under appropriate $\mathrm{Rh}(\mathrm{III})$-catalyzed conditions. We now report synthesis of indolines via this type of coupling, and the indoline motif has been widely found in natural products and pharmaceuticals [54-56].

\section{Experimental}

\subsection{General}

All chemicals were from commercial sources and were used as received unless otherwise noted. All reactions were carried out using Schlenk techniques or in an $\mathrm{N}_{2}$-filled glovebox. Column chromatography was carried out on silica gel (300-400 mesh) using triethylamine/petroleum ether or ethyl acetate (EA)/petroleum ether (PE). NMR Spectra were recorded on a Bruker $400 \mathrm{MHz}$ NMR spectrometer in the solvents indicated. The chemical shift is given in dimensionless $\delta$ values and is frequency referenced relative to TMS in ${ }^{1} \mathrm{H}$ and ${ }^{13} \mathrm{C}$ NMR spectroscopy. The ratio of trans and cis was determined on the basis of ${ }^{1} \mathrm{H}$ NMR analysis. High resolution mass spectra were obtained on an Agilent Q-TOF 6540.

Diphenylacetylene and 4-octyne were obtained from commercial sources. Other nitrones [50] and diarylacetylenes [57] were prepared according to literature reports and the NMR data agree with those in the literature reports.

\subsection{General procedure for the synthesis of compounds 3}

Nitrones $(0.2 \mathrm{mmol})$, diarylacetylenes $(0.3 \mathrm{mmol})$, [Cp* $\mathrm{RhCl}_{2}$ 2 (4 mol\%), AgSbF6 (16 mol\%), PivOH (2.0 equiv) and ethyl acetate $(2 \mathrm{~mL})$ were charged into the sealed tube. The reaction mixture was stirred at $80{ }^{\circ} \mathrm{C}$ for $15 \mathrm{~h}$. After cooled to room temperature, the solvent was removed under reduced pressure and the residue was purified by silica gel chromatography using triethylamine/PE or PE/EA to afford compounds 3.

\subsection{Spectral data for products}

3aa. ${ }^{1} \mathrm{H}$ NMR (400 MHz, $\left.\mathrm{CDCl}_{3}\right) \delta 7.63-7.51(\mathrm{~m}, 2 \mathrm{H}), 7.44-$ $7.35(\mathrm{~m}, 1 \mathrm{H}), 7.30-7.20(\mathrm{~m}, 2 \mathrm{H}), 7.17(\mathrm{td}, J=7.7,1.2 \mathrm{~Hz}, 1 \mathrm{H})$, $7.12-6.95(\mathrm{~m}, 9 \mathrm{H}), 6.82(\mathrm{~d}, J=7.6 \mathrm{~Hz}, 1 \mathrm{H}), 6.73-6.60(\mathrm{~m}, 3 \mathrm{H})$, $6.17(\mathrm{~s}, 1 \mathrm{H}), 4.28(\mathrm{~s}, 1 \mathrm{H}) .{ }^{13} \mathrm{C} \mathrm{NMR}\left(100 \mathrm{MHz}, \mathrm{CDCl}_{3}\right) \delta 197.5$, $152.2,140.0,138.4,137.2,132.2,131.0,129.4,128.6,128.4$, 128.1, 128.0, 127.99, 127.7, 127.3, 127.1, 126.3, 119.0, 109.8, 72.3, 70.3. HRMS: $m / z$ : $[\mathrm{M}+\mathrm{H}]^{+}$calculated for $\mathrm{C}_{27} \mathrm{H}_{21} \mathrm{NO}$ : 376.1696, Found: 376.1696.

3aa'. ${ }^{1} \mathrm{H}$ NMR (400 MHz, $\left.\mathrm{CDCl}_{3}\right) \delta 7.68-7.60(\mathrm{~m}, 2 \mathrm{H}), 7.57-$ $7.50(\mathrm{~m}, 1 \mathrm{H}), 7.32(\mathrm{t}, J=7.6 \mathrm{~Hz}, 2 \mathrm{H}), 7.27-7.21(\mathrm{~m}, 4 \mathrm{H}), 7.07$ $(\mathrm{m}, 3 \mathrm{H}), 7.00(\mathrm{~m}, 1 \mathrm{H}), 6.88(\mathrm{t}, J=7.7 \mathrm{~Hz}, 2 \mathrm{H}), 6.85-6.78(\mathrm{~m}$, 3H), $6.67(\mathrm{~d}, J=7.7 \mathrm{~Hz}, 1 \mathrm{H}), 5.50(\mathrm{~s}, 1 \mathrm{H}), 4.26(\mathrm{~s}, 1 \mathrm{H}) .{ }^{13} \mathrm{C} \mathrm{NMR}$ $\left(100 \mathrm{MHz}_{\mathrm{CDCl}}\right) \delta 1 \mathrm{197.6}, 148.7,145.1,141.2,138.1,131.9$, 131.8, 129.7, 129.6, 128.4, 128.3, 128.2, 128.0, 127.9, 127.5, 127.45, 124.8, 119.74, 109.8, 71.4, 70.3. HRMS: $m / z$ : $[\mathrm{M}+\mathrm{H}]^{+}$ calculated for $\mathrm{C}_{27} \mathrm{H}_{21} \mathrm{NO}$ : 376.1696, Found: 376.1697 .

3ba. ${ }^{1} \mathrm{H}$ NMR (400 MHz, $\left.\mathrm{CDCl}_{3}\right) \delta 7.48(\mathrm{~d}, J=7.4 \mathrm{~Hz}, 2 \mathrm{H})$, $7.29(\mathrm{t}, J=7.4 \mathrm{~Hz}, 1 \mathrm{H}), 7.13(\mathrm{t}, J=7.9 \mathrm{~Hz}, 2 \mathrm{H}), 7.00-6.86(\mathrm{~m}$, $9 \mathrm{H}), 6.73(\mathrm{~s}, 1 \mathrm{H}), 6.63(\mathrm{~d}, J=7.9 \mathrm{~Hz}, 1 \mathrm{H}), 6.57(\mathrm{~d}, J=7.3 \mathrm{~Hz}, 2 \mathrm{H})$, $6.05(\mathrm{~s}, 1 \mathrm{H}), 4.03(\mathrm{~s}, 1 \mathrm{H}), 2.02(\mathrm{~s}, 3 \mathrm{H}) .{ }^{13} \mathrm{C}$ NMR $(100 \mathrm{MHz}$, $\left.\mathrm{CDCl}_{3}\right) \delta 197.6,149.8,140.0,138.3,137.1,132.0,130.8,129.7$, 128.5, 128.3, 128.27, 128.1, 127.9, 127.8 127.5, 127.1, 126.9, 126.6, 109.7, 72.3, 70.5, 21.1. HRMS: $m / z$ : $[\mathrm{M}+\mathrm{H}]^{+}$calculated for $\mathrm{C}_{28} \mathrm{H}_{23} \mathrm{NO}: 390.1852$, Found: 390.1855 .

3ca. ${ }^{1} \mathrm{H}$ NMR (400 MHz, $\left.\mathrm{CDCl}_{3}\right) \delta 7.53(\mathrm{~d}, J=7.9 \mathrm{~Hz}, 2 \mathrm{H}), 7.40$ $(\mathrm{t}, J=7.4 \mathrm{~Hz}, 1 \mathrm{H}), 7.23(\mathrm{~m}, 2 \mathrm{H}), 7.11-6.97(\mathrm{~m}, 8 \mathrm{H}), 6.87(\mathrm{td}, J=$ 8.7, $2.5 \mathrm{~Hz}, 1 \mathrm{H}), 6.72(\mathrm{~m}, 2 \mathrm{H}), 6.62(\mathrm{~d}, J=7.7 \mathrm{~Hz}, 2 \mathrm{H}), 6.16(\mathrm{~s}$, 1H), $4.14(\mathrm{~s}, 1 \mathrm{H}) .{ }^{13} \mathrm{C} \mathrm{NMR}\left(100 \mathrm{MHz}, \mathrm{CDCl}_{3}\right) \delta 196.9,156.6(\mathrm{~d}$, $\left.J_{C-F}=233.9 \mathrm{~Hz}\right), 148.2,148.2,139.4,137.7,136.7,132.4,130.7$, 128.3, 128.25, 128.1 (d, $\left.J_{C-F}=1.4 \mathrm{~Hz}\right), 127.7$ (d, $J_{C-F}=7.8 \mathrm{~Hz}$ ), 127.5, 127.3, 127.2, 115.7 (d, JC-F $=23.3 \mathrm{~Hz}), 115.0$ (d, JC-F $=25$ $\mathrm{Hz}), 110.0\left(\mathrm{~d}, J_{C-F}=8.1 \mathrm{~Hz}\right), 72.1,70.8$. HRMS: $m / z$ : $[\mathrm{M}+\mathrm{H}]^{+}$calculated for $\mathrm{C}_{27} \mathrm{H}_{20}$ FNO: 394.1602, Found: 394.1604 .

3da. ${ }^{1} \mathrm{H}$ NMR (400 MHz, $\left.\mathrm{CDCl}_{3}\right) \delta 7.57-7.48(\mathrm{~m}, 2 \mathrm{H}), 7.42-$ $7.37(\mathrm{~m}, 1 \mathrm{H}), 7.23(\mathrm{~m}, 2 \mathrm{H}), 7.08(\mathrm{~m}, 2 \mathrm{H}), 7.03-6.93(\mathrm{~m}, 8 \mathrm{H})$, $6.71(\mathrm{~d}, J=8.4 \mathrm{~Hz}, 1 \mathrm{H}), 6.67-6.61(\mathrm{~m}, 2 \mathrm{H}), 6.16(\mathrm{~s}, 1 \mathrm{H}), 4.26(\mathrm{~s}$, 1H). ${ }^{13} \mathrm{C}$ NMR (100 MHz, $\left.\mathrm{CDCl}_{3}\right) \delta 196.9,150.7,139.3,137.6$, $136.5,132.4,130.7,129.2,128.3,128.2,128.1,128.02,128.0$, $127.9,127.6,127.3,127.2,123.2,110.4,72.2,70.5$. HRMS: m/z: $[\mathrm{M}+\mathrm{H}]^{+}$calculated for $\mathrm{C}_{27} \mathrm{H}_{20} \mathrm{ClNO}: 410.1306$, Found: 410.1302 .

3ea. ${ }^{1} \mathrm{H}$ NMR (400 MHz, $\left.\mathrm{CDCl}_{3}\right) \delta 7.43(\mathrm{~d}, J=7.4 \mathrm{~Hz}, 2 \mathrm{H})$, $7.32(\mathrm{t}, J=7.4 \mathrm{~Hz}, 1 \mathrm{H}), 7.18-7.12(\mathrm{~m}, 3 \mathrm{H}), 6.99(\mathrm{t}, J=4.9 \mathrm{~Hz}$, $2 \mathrm{H}), 6.96-6.86(\mathrm{~m}, 7 \mathrm{H}), 6.58(\mathrm{t}, J=8.3 \mathrm{~Hz}, 3 \mathrm{H}), 6.08(\mathrm{~s}, 1 \mathrm{H})$, 4.20 (s, 1H). ${ }^{13} \mathrm{C}$ NMR $\left(100 \mathrm{MHz}, \mathrm{CDCl}_{3}\right) \delta 196.9,151.1,139.2$, 137.6, 136.5, 132.4, 132.0, 130.7, 130.65, 128.4, 128.3, 128.13, 128.1, 128.0, 127.6, 127.3, 127.2, 110.9, 110.1, 72.2, 70.4. HRMS: $m / z$ : $[\mathrm{M}+\mathrm{H}]^{+}$calculated for $\mathrm{C}_{27} \mathrm{H}_{20} \mathrm{BrNO}$ : 454.0801, Found: 454.0801 .

3fa. ${ }^{1} \mathrm{H}$ NMR (400 MHz, $\left.\mathrm{CDCl}_{3}\right) \delta 7.79(\mathrm{dd}, J=8.3,1.4 \mathrm{~Hz}$, $1 \mathrm{H}), 7.64(\mathrm{~s}, 1 \mathrm{H}), 7.47$ (d, J = 7.5 Hz, 2H), 7.30 (t, J = 7.4 Hz, 1H), $7.15(\mathrm{~m}, 2 \mathrm{H}), 7.01-6.83(\mathrm{~m}, 8 \mathrm{H}), 6.63(\mathrm{t}, J=8.0 \mathrm{~Hz}, 3 \mathrm{H}), 6.14(\mathrm{~s}$, $1 \mathrm{H}), 4.67(\mathrm{~s}, 1 \mathrm{H}), 4.09(\mathrm{~m}, 2 \mathrm{H}), 1.14(\mathrm{t}, J=7.1 \mathrm{~Hz}, 3 \mathrm{H}) .{ }^{13} \mathrm{C} \mathrm{NMR}$ $\left(100 \mathrm{MHz} \mathrm{CDCl}_{3}\right) \delta 197.1,166.4,155.9,139.1,137.7,136.4$, $132.3,132.1,130.7,130.2,128.3,128.2,128.0,127.9,127.6$, 127.4, 127.2, 125.4, 120.5, 108.1, 71.8, 70.2, 60.2, 14.3. HRMS: 
$m / z:[\mathrm{M}+\mathrm{H}]^{+}$calculated for $\mathrm{C}_{30} \mathrm{H}_{25} \mathrm{NO}_{3}:$ 448.1907, Found: 448.1906.

3ga. ${ }^{1} \mathrm{H}$ NMR (400 MHz, $\left.\mathrm{CDCl}_{3}\right) \delta 7.55(\mathrm{~d}, J=7.4 \mathrm{~Hz}, 2 \mathrm{H})$, $7.37(\mathrm{t}, J=7.4 \mathrm{~Hz}, 1 \mathrm{H}), 7.22(\mathrm{~m} \mathrm{2H}), 7.08-6.94(\mathrm{~m}, 8 \mathrm{H}), 6.87$ (d, $J=7.8 \mathrm{~Hz}, 1 \mathrm{H}), 6.72-6.61(\mathrm{~m}, 3 \mathrm{H}), 6.46(\mathrm{~d}, J=7.8 \mathrm{~Hz}, 1 \mathrm{H}), 6.13$ $(\mathrm{s}, 1 \mathrm{H}), 4.19$ (s, 1H), 2.29 (s, 3H). ${ }^{13} \mathrm{C} \mathrm{NMR}\left(100 \mathrm{MHz}, \mathrm{CDCl}_{3}\right) \delta$ 197.3, 152.3, 140.0, 139.4, 138.4, 136.9, 132.0, 130.8, 128.4, 128.2, 127.9, 127.8, 127.6, 127.5, 127.1, 126.9, 123.1, 119.8, 110.5, 71.9, 70.2, 21.6. HRMS: $\mathrm{m} / \mathrm{z}:[\mathrm{M}+\mathrm{H}]^{+}$calculated for $\mathrm{C}_{28} \mathrm{H}_{23} \mathrm{NO}$ : 390.1852, Found: 390.1852.

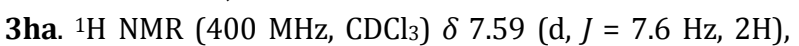
$7.37(\mathrm{t}, J=7.4 \mathrm{~Hz}, 1 \mathrm{H}), 7.21(\mathrm{t}, J=7.8 \mathrm{~Hz}, 2 \mathrm{H}), 7.00(\mathrm{~m}, 8 \mathrm{H}), 6.90$ (d, $J=8.1 \mathrm{~Hz}, 1 \mathrm{H}), 6.86(\mathrm{~d}, J=1.4 \mathrm{~Hz}, 1 \mathrm{H}) 6.68(\mathrm{dd}, J=8.1,1.5$ $\mathrm{Hz}, 1 \mathrm{H}), 6.60$ (d, $J=7.4 \mathrm{~Hz}, 2 \mathrm{H}), 6.17(\mathrm{~s}, 1 \mathrm{H}), 4.18(\mathrm{~s}, 1 \mathrm{H}), 1.30$ (s, 9H). ${ }^{13} \mathrm{C}$ NMR $\left(100 \mathrm{MHz}, \mathrm{CDCl}_{3}\right) \delta 197.3,152.8,151.9,139.9$, 138.7, 137.0, 132.1, 131.1, 128.5, 127.9, 127.8, 127.4, 127.1, 126.9, 126.88, 123.6, 116.3, 107.2, 71.7, 70.4, 34.8, 31.5. HRMS: $m / z:[\mathrm{M}+\mathrm{H}]^{+}$calculated for $\mathrm{C}_{28} \mathrm{H}_{23} \mathrm{NO}: 432.2322$, Found: 432.2327.

3ia. ${ }^{1} \mathrm{H}$ NMR (400 MHz, $\left.\mathrm{CDCl}_{3}\right) \delta 7.58(\mathrm{~m}, 4 \mathrm{H}), 7.40(\mathrm{q}, J=7.1$ $\mathrm{Hz}, 3 \mathrm{H}), 7.32$ (m, 1H), $7.23(\mathrm{~m}, 2 \mathrm{H}), 7.10-6.96(\mathrm{~m}, 10 \mathrm{H}), 6.88$ (dd, $J=7.9,1.6 \mathrm{~Hz}, 1 \mathrm{H}), 6.77-6.67(\mathrm{~m}, 2 \mathrm{H}), 6.20(\mathrm{~s}, 1 \mathrm{H}), 4.32$ (s, 1H). ${ }^{13} \mathrm{C}$ NMR $\left(100 \mathrm{MHz}, \mathrm{CDCl}_{3}\right) \delta 197.2,152.6,142.5,141.1$, $139.8,138.3,136.9,132.1,130.9,128.7,128.4,128.3,128.1$, 128.0, 127.9, 127.6, 127.4, 127.2, 127.1, 127.06, 125.3, 118.1, 108.3, 72.0, 70.4. HRMS: $m / z$ : $[\mathrm{M}+\mathrm{H}]^{+}$calculated for $\mathrm{C}_{28} \mathrm{H}_{24} \mathrm{NO}$ : 452.2009, Found: 452.2006.

3ja. ${ }^{1} \mathrm{H}$ NMR (400 MHz, $\left.\mathrm{CDCl}_{3}\right) \delta 7.54(\mathrm{~d}, J=8.2 \mathrm{~Hz}, 2 \mathrm{H}), 7.37$ $(\mathrm{t}, J=7.4 \mathrm{~Hz}, 1 \mathrm{H}), 7.21(\mathrm{t}, J=7.8 \mathrm{~Hz}, 2 \mathrm{H}), 7.14(\mathrm{t}, J=7.5 \mathrm{~Hz}, 1 \mathrm{H})$, $7.07(\mathrm{t}, J=7.2 \mathrm{~Hz}, 1 \mathrm{H}), 7.00(\mathrm{t}, J=7.2 \mathrm{~Hz}, 3 \mathrm{H}), 6.87(\mathrm{~d}, J=7.8 \mathrm{~Hz}$, $2 \mathrm{H}), 6.79(\mathrm{~d}, J=7.3 \mathrm{~Hz}, 3 \mathrm{H}), 6.66(\mathrm{~d}, J=7.5,2 \mathrm{H}), 6.62$ (d, $J=7.5$, $1 \mathrm{H}), 6.11(\mathrm{~s}, 1 \mathrm{H}), 4.19(\mathrm{~s}, 1 \mathrm{H}), 2.17(\mathrm{~s}, 3 \mathrm{H}) .{ }^{13} \mathrm{C}$ NMR $(100 \mathrm{MHz}$, $\left.\mathrm{CDCl}_{3}\right) \delta 197.5,152.1,138.3,137.0,136.8,136.6,132.0,130.8$, 129.2, 128.5, 128.2, 128.16, 127.9, 127.8, 127.78, 127.0, 126.3, 118.8, 109.7, 72.0, 70.1, 21.1. HRMS: $m / z:[\mathrm{M}+\mathrm{H}]^{+}$calculated for $\mathrm{C}_{28} \mathrm{H}_{23} \mathrm{NO}$ : 390.1852, Found: 390.1853.

3ka. ${ }^{1} \mathrm{H}$ NMR $\left(400 \mathrm{MHz}, \mathrm{CDCl}_{3}\right) \delta 7.47(\mathrm{~d}, J=7.8 \mathrm{~Hz}, 2 \mathrm{H})$, $7.29(\mathrm{t}, J=7.3 \mathrm{~Hz}, 1 \mathrm{H}), 7.13(\mathrm{~m}, 2 \mathrm{H}), 7.06(\mathrm{t}, J=7.6 \mathrm{~Hz}, 1 \mathrm{H}), 7.02$ $-6.96(\mathrm{~m}, 1 \mathrm{H}), 6.92(\mathrm{~m}, 3 \mathrm{H}), 6.83(\mathrm{~d}, J=8.3 \mathrm{~Hz}, 2 \mathrm{H}), 6.71(\mathrm{~d}, J=$ $7.8 \mathrm{~Hz}, 1 \mathrm{H}), 6.62-6.49(\mathrm{~m}, 3 \mathrm{H}), 6.45(\mathrm{~d}, J=8.5 \mathrm{~Hz}, 2 \mathrm{H}), 6.02(\mathrm{~s}$, $1 \mathrm{H}), 4.07$ (s, 1H), $3.58(\mathrm{~s}, 3 \mathrm{H}) .{ }^{13} \mathrm{C}$ NMR $\left(100 \mathrm{MHz}, \mathrm{CDCl}_{3}\right) \delta$ 197.6, 158.8, 152.0, 138.4, 137.1, 132.0, 131.7, 130.8, 129.4, $129.2,128.5,128.0,127.8,127.7,127.0,126.4,118.8,112.9$, 109.7, 71.9, 69.8, 55.2. HRMS: $m / z:[\mathrm{M}+\mathrm{H}]^{+}$calculated for $\mathrm{C}_{28} \mathrm{H}_{23} \mathrm{NO}_{2}: 406.1802$, Found: 406.1805.

3la. ${ }^{1} \mathrm{H}$ NMR (400 MHz, $\left.\mathrm{CDCl}_{3}\right) \delta 7.56(\mathrm{~d}, J=8.0 \mathrm{~Hz}, 2 \mathrm{H}), 7.39$ (t, $J=7.4 \mathrm{~Hz}, 1 \mathrm{H}), 7.26-7.19(\mathrm{~m}, 2 \mathrm{H}), 7.16(\mathrm{t}, J=7.3 \mathrm{~Hz}, 1 \mathrm{H})$, $7.09(\mathrm{t}, J=7.3 \mathrm{~Hz}, 1 \mathrm{H}), 7.05-6.93(\mathrm{~m}, 7 \mathrm{H}), 6.80(\mathrm{~d}, J=7.8 \mathrm{~Hz}$, $1 \mathrm{H}), 6.65(\mathrm{t}, J=7.5 \mathrm{~Hz}, 1 \mathrm{H}), 6.59(\mathrm{~d}, J=7.5 \mathrm{~Hz}, 2 \mathrm{H}), 6.13(\mathrm{~s}, 1 \mathrm{H})$, 4.22 (s, 1H). ${ }^{13} \mathrm{C}$ NMR (100 MHz, $\left.\mathrm{CDCl}_{3}\right) \delta 197.4,151.7,138.2$, $138.0,136.9,132.9,132.3,130.9,129.7,129.3,128.4,128.1$, 127.9, 127.6, 127.57, 127.2, 126.3, 119.1, 109.9, 71.9, 69.5. HRMS: $m / z$ : $[\mathrm{M}+\mathrm{H}]^{+}$calculated for $\mathrm{C}_{27} \mathrm{H}_{20} \mathrm{ClNO}$ : 410.1306, Found: 410.1309 .

3ma. ${ }^{1} \mathrm{H}$ NMR (400 MHz, $\left.\mathrm{CDCl}_{3}\right) \delta 7.57(\mathrm{~d}, J=7.6 \mathrm{~Hz}, 2 \mathrm{H})$, $7.41(\mathrm{t}, J=7.4 \mathrm{~Hz}, 1 \mathrm{H}), 7.28-7.21(\mathrm{~m}, 2 \mathrm{H}), 7.18(\mathrm{~m}, 1 \mathrm{H}), 7.11(\mathrm{t}$, $J=8.5 \mathrm{~Hz}, 3 \mathrm{H}), 7.02(\mathrm{~m}, 3 \mathrm{H}), 6.92(\mathrm{~d}, J=8.2 \mathrm{~Hz}, 2 \mathrm{H}), 6.82$ (d, $J=$
$7.8 \mathrm{~Hz}, 1 \mathrm{H}), 6.67$ (t, J = 7.5 Hz, 1H), $6.61(\mathrm{~d}, J=7.4 \mathrm{~Hz}, 2 \mathrm{H}), 6.13$ (s, 1H), $4.24(\mathrm{~s}, 1 \mathrm{H}) .{ }^{13} \mathrm{C} \mathrm{NMR}\left(100 \mathrm{MHz}, \mathrm{CDCl}_{3}\right) \delta 197.4,151.6$, 138.8, 138.0, 136.9, 132.3, 130.9, 130.5, 130.1, 129.3, 128.4, 128.1, 127.9, 127.5, 127.3, 126.3, 121.1, 119.2, 109.9, 71.9, 69.6. HRMS: $m / z$ : $[\mathrm{M}+\mathrm{H}]^{+}$calculated for $\mathrm{C}_{27} \mathrm{H}_{20} \mathrm{BrNO}$ : 454.0801, Found: 454.0802 .

3na. ${ }^{1} \mathrm{H} \mathrm{NMR}\left(400 \mathrm{MHz}, \mathrm{CDCl}_{3}\right) \delta 7.61-7.56(\mathrm{~m}, 2 \mathrm{H}), 7.40(\mathrm{t}$, $J=7.4 \mathrm{~Hz}, 1 \mathrm{H}), 7.24(\mathrm{~m}, 2 \mathrm{H}), 7.19-7.11(\mathrm{~m}, 2 \mathrm{H}), 7.07(\mathrm{t}, J=7.4$ $\mathrm{Hz}, 2 \mathrm{H}), 7.02$ (d, $J=7.6 \mathrm{~Hz}, 1 \mathrm{H}), 6.94$ (dd, $J=5.0,1.0 \mathrm{~Hz}, 1 \mathrm{H}$ ), $6.84(\mathrm{~m}, 2 \mathrm{H}), 6.74(\mathrm{~m}, 3 \mathrm{H}), 6.68(\mathrm{td}, J=7.6,0.9 \mathrm{~Hz}, 1 \mathrm{H}), 6.37(\mathrm{~s}$, $1 \mathrm{H}), 4.36(\mathrm{~s}, 1 \mathrm{H}) .{ }^{13} \mathrm{C} \mathrm{NMR}\left(100 \mathrm{MHz}, \mathrm{CDCl}_{3}\right) \delta 197.2,151.2$, $143.6,137.9,136.9,132.3,130.9,129.2,128.1,128.0,127.9$, 127.6, 127.3, 126.4, 126.1, 125.9, 124.7, 119.4, 110.3, 71.6, 66.7. HRMS: $m / z:[\mathrm{M}+\mathrm{H}]^{+}$calculated for $\mathrm{C}_{25} \mathrm{H}_{20} \mathrm{NOS}$ : 382.1260, Found: 382.1258

3ab. ${ }^{1} \mathrm{H}$ NMR (400 MHz, $\left.\mathrm{CDCl}_{3}\right) \delta 7.50(\mathrm{~d}, J=8.3 \mathrm{~Hz}, 2 \mathrm{H})$, $7.13(\mathrm{td}, J=7.8,1.0 \mathrm{~Hz}, 1 \mathrm{H}), 7.06-6.94(\mathrm{~m}, 8 \mathrm{H}), 6.78(\mathrm{dd}, J=$ 8.0, $1.8 \mathrm{~Hz}, 3 \mathrm{H}), 6.64(\mathrm{td}, J=7.6,0.8 \mathrm{~Hz}, 1 \mathrm{H}), 6.49(\mathrm{~d}, J=8.1 \mathrm{~Hz}$, $2 \mathrm{H}), 6.13(\mathrm{~s}, 1 \mathrm{H}), 4.33(\mathrm{~s}, 1 \mathrm{H}), 2.29(\mathrm{~s}, 3 \mathrm{H}), 2.18(\mathrm{~s}, 3 \mathrm{H}) .{ }^{13} \mathrm{C} \mathrm{NMR}$ $\left(100 \mathrm{MHz} \mathrm{CDCl}_{3}\right) \delta 197.0,152.0,142.7,140.0,136.5,135.4$, 134.2, 131.2, 129.1, 128.6, 128.55, 128.4, 128.3, 127.7, 127.4, 127.1, 126.8, 118.8, 109.7, 71.7, 70.2, 21.5, 21.0. HRMS: $m / z$ : $[\mathrm{M}+\mathrm{H}]^{+}$calculated for $\mathrm{C}_{29} \mathrm{H}_{25} \mathrm{NO}: 404.2009$, Found: 404.2005.

3ac. ${ }^{1} \mathrm{H}$ NMR $\left(400 \mathrm{MHz}, \mathrm{CDCl}_{3}\right) \delta 7.64(\mathrm{~m}, 2 \mathrm{H}), 7.17(\mathrm{~m}, 1 \mathrm{H})$, $7.10-6.98(\mathrm{~m}, 6 \mathrm{H}), 6.81(\mathrm{~d}, J=7.8 \mathrm{~Hz}, 1 \mathrm{H}), 6.73(\mathrm{~d}, J=9.0 \mathrm{~Hz}$, $2 \mathrm{H}), 6.67(\mathrm{t}, J=7.5 \mathrm{~Hz}, 1 \mathrm{H}), 6.50(\mathrm{q}, J=8.9 \mathrm{~Hz}, 4 \mathrm{H}), 6.17(\mathrm{~s}, 1 \mathrm{H})$, 4.23 (s, 1H), 3.79 (s, 3H), 3.69 (s, 3H). ${ }^{13} \mathrm{C}$ NMR (100 MHz, $\left.\mathrm{CDCl}_{3}\right) \delta 196.0,162.6,158.4,151.8,139.9,133.5,131.0,129.5$, $129.4,129.0,128.4,127.5,127.4,127.3,127.1,118.8,113.2$, 113.1, 109.7, 71.0, 70.2, 55.3, 55.2. HRMS: $m / z$ : $[\mathrm{M}+\mathrm{H}]^{+}$calculated for $\mathrm{C}_{29} \mathrm{H}_{25} \mathrm{NO}_{3}: 436.1907$, Found: 436.1907.

3ad. ${ }^{1} \mathrm{H}$ NMR $\left(400 \mathrm{MHz}, \mathrm{CDCl}_{3}\right) \delta 7.62(\mathrm{~m}, 2 \mathrm{H}), 7.18(\mathrm{td}, J=$ 7.8, $1.1 \mathrm{~Hz}, 1 \mathrm{H}), 7.08-6.89(\mathrm{~m}, 8 \mathrm{H}), 6.82(\mathrm{~d}, J=7.7 \mathrm{~Hz}, 1 \mathrm{H}), 6.74$ - $6.64(\mathrm{~m}, 3 \mathrm{H}), 6.59-6.51(\mathrm{~m}, 2 \mathrm{H}), 6.15(\mathrm{~s}, 1 \mathrm{H}), 4.25(\mathrm{~s}, 1 \mathrm{H}) .{ }^{13} \mathrm{C}$ NMR (100 MHz, CDCl $) \delta 195.5,164.9\left(\mathrm{~d}, J_{C-F}=253.4 \mathrm{~Hz}\right), 161.8$ $\left(\mathrm{d}, J_{C-F}=245.7 \mathrm{~Hz}\right), 151.9,139.3,134.1\left(\mathrm{~d}, J_{C-F}=3.3 \mathrm{~Hz}\right), 133.6$ $\left(\mathrm{d}, J_{C-F}=9.0 \mathrm{~Hz}\right), 132.8\left(\mathrm{~d}, J_{C-F}=3.2 \mathrm{~Hz}\right), 130.0\left(\mathrm{~d}, J_{C-F}=8.0 \mathrm{~Hz}\right.$ ), $129.5,128.2,127.7,127.4,127.2,126.2,119.1,115.1$ (d, $J_{C-F}=$ $21.5 \mathrm{~Hz}$ ), 114.9 (d, $J_{C-F}=21.3 \mathrm{~Hz}$ ), 109.9, 71.2, 70.2. HRMS: $m / z$ : $[\mathrm{M}+\mathrm{H}]^{+}$calculated for $\mathrm{C}_{27} \mathrm{H}_{19} \mathrm{~F}_{2} \mathrm{NO}: 412.1507$, Found: 412.1506 .

3ae. ${ }^{1} \mathrm{H}$ NMR ( $\left.400 \mathrm{MHz}, \mathrm{CDCl}_{3}\right) \delta 7.43(\mathrm{~m}, 2 \mathrm{H}), 7.14(\mathrm{t}, J=6.0$ $\mathrm{Hz}, 2 \mathrm{H}), 7.09(\mathrm{t}, J=7.7 \mathrm{~Hz}, 1 \mathrm{H}), 6.99-6.84(\mathrm{~m}, 8 \mathrm{H}), 6.72(\mathrm{~d}, J=$ $7.8 \mathrm{~Hz}, 1 \mathrm{H}), 6.59(\mathrm{t}, J=7.6 \mathrm{~Hz}, 1 \mathrm{H}), 6.42(\mathrm{dd}, J=8.5,1.4 \mathrm{~Hz}, 2 \mathrm{H})$, $6.05(\mathrm{~s}, 1 \mathrm{H}), 4.15$ (s, 1H). ${ }^{13} \mathrm{C}$ NMR $\left(100 \mathrm{MHz}, \mathrm{CDCl}_{3}\right) \delta 195.6$, 151.9, 139.1, 138.8, 136.8, 134.9, 133.2, 132.3, 129.7, 129.6, 129.1, 128.3, 128.2, 127.7, 127.6, 127.2, 125.8, 119.1, 110.0, 71.4, 70.2. HRMS: $m / z$ : $[\mathrm{M}+\mathrm{H}]^{+}$calculated for $\mathrm{C}_{27} \mathrm{H}_{19} \mathrm{Cl}_{2} \mathrm{NO}$ : 444.0916, Found: 444.0912.

3af. ${ }^{1} \mathrm{H}$ NMR (400 MHz, $\left.\mathrm{CDCl}_{3}\right) \delta 7.41(\mathrm{q}, J=8.7 \mathrm{~Hz}, 4 \mathrm{H}), 7.18$ (t, $J=7.6 \mathrm{~Hz}, 1 \mathrm{H}), 7.11(\mathrm{~d}, J=8.5 \mathrm{~Hz}, 1 \mathrm{H}), 7.08-6.97(\mathrm{~m}, 5 \mathrm{H})$, $6.94(\mathrm{~d}, J=7.6 \mathrm{~Hz}, 1 \mathrm{H}), 6.81(\mathrm{~d}, J=7.8 \mathrm{~Hz}, 1 \mathrm{H}), 6.67$ (t, $J=7.5 \mathrm{~Hz}$, $1 \mathrm{H}), 6.44(\mathrm{~d}, J=8.5 \mathrm{~Hz}, 2 \mathrm{H}), 6.13(\mathrm{~s}, 1 \mathrm{H}), 4.23(\mathrm{~s}, 1 \mathrm{H}) .{ }^{13} \mathrm{C} \mathrm{NMR}$ $\left(100 \mathrm{MHz} \mathrm{CDCl}_{3}\right) \delta 195.8,151.9,139.0,137.3,135.3,132.4$, 131.3, 131.1, 130.1, 129.6, 128.1, 127.8, 127.6, 127.6, 127.2, 125.6, 121.4, 119.1, 110.0, 71.4, 70.1. HRMS: $m / z:[\mathrm{M}+\mathrm{H}]^{+}$calculated for $\mathrm{C}_{27} \mathrm{H}_{19} \mathrm{Br}_{2} \mathrm{NO}$ : 531.9906, Found: 531.9906.

3ag. ${ }^{1} \mathrm{H}$ NMR (400 MHz, $\left.\mathrm{CDCl}_{3}\right) \delta 7.50(\mathrm{~s}, 1 \mathrm{H}), 7.25-7.11(\mathrm{~m}$, 
3H), $7.05(\mathrm{t}, J=7.7 \mathrm{~Hz}, 1 \mathrm{H}), 7.03-6.95(\mathrm{~m}, 6 \mathrm{H}), 6.93-6.83(\mathrm{~m}$, 2H), $6.79(\mathrm{~d}, J=7.8 \mathrm{~Hz}, 1 \mathrm{H}), 6.64(\mathrm{t}, J=7.5 \mathrm{~Hz}, 1 \mathrm{H}), 6.49(\mathrm{~d}, J=$ $7.4 \mathrm{~Hz}, 1 \mathrm{H}), 6.40(\mathrm{~s}, 1 \mathrm{H}), 6.12(\mathrm{~s}, 1 \mathrm{H}), 4.25(\mathrm{~s}, 1 \mathrm{H}), 2.24(\mathrm{~s}, 3 \mathrm{H})$, 2.01 (s, 3H). ${ }^{13} \mathrm{C}$ NMR (100 MHz, $\left.\mathrm{CDCl}_{3}\right) \delta 197.7,152.1,140.0$, $138.1,137.7,137.5,137.1,132.8,131.3,129.2,129.1,128.3$, $128.1,128.0,127.8,127.6,127.5,127.4,127.1,126.2,125.4$, 118.8, 109.6, 72.2, 70.1, 21.4, 21.2. HRMS: $m / z$ : $[\mathrm{M}+\mathrm{H}]^{+}$calculated for $\mathrm{C}_{29} \mathrm{H}_{26} \mathrm{NO}: 404.2009$, Found: 404.2009.

3ah. ${ }^{1} \mathrm{H}$ NMR (400 MHz, $\left.\mathrm{CDCl}_{3}\right) \delta 7.32-7.22(\mathrm{~m}, 2 \mathrm{H}), 7.21$ $7.15(\mathrm{~m}, 2 \mathrm{H}), 7.14-7.08(\mathrm{~m}, 1 \mathrm{H}), 7.05(\mathrm{~m}, 1 \mathrm{H}), 6.97(\mathrm{~m}, 6 \mathrm{H})$, $6.83-6.75(\mathrm{~m}, 2 \mathrm{H}), 6.67(\mathrm{t}, J=7.5 \mathrm{~Hz}, 1 \mathrm{H}), 6.41(\mathrm{~m}, 2 \mathrm{H}), 6.12(\mathrm{~s}$, $1 \mathrm{H}), 4.30(\mathrm{~s}, 1 \mathrm{H}) .{ }^{13} \mathrm{C} \mathrm{NMR}\left(100 \mathrm{MHz}, \mathrm{CDCl}_{3}\right) \delta 195.4\left(\mathrm{~d}, J_{C-F}=\right.$ $1.9 \mathrm{~Hz}$ ), 162.7 (d, $J_{C-F}=247.5 \mathrm{~Hz}$ ), 162.1 (d, $J_{C-F}=248.2 \mathrm{~Hz}$ ), 152.0, $140.6\left(\mathrm{~d}, J_{C-F}=7.2 \mathrm{~Hz}\right), 139.2,138.8\left(\mathrm{~d}, J_{C-F}=6.3 \mathrm{~Hz}\right.$ ), 129.7, 129.5 (d, JC-F $=2.5 \mathrm{~Hz}), 129.4\left(\mathrm{~d}, J_{C-F}=3.2 \mathrm{~Hz}\right), 128.0$, $127.7,127.6,127.6,126.5\left(\mathrm{~d}, J_{C-F}=3.1 \mathrm{~Hz}\right), 125.0,124.3\left(\mathrm{~d}, J_{C-F}=\right.$ $2.7 \mathrm{~Hz}), 119.3\left(\mathrm{~d}, J_{C-F}=21.4 \mathrm{~Hz}\right), 119.1,117.4\left(\mathrm{~d}, J_{C-F}=23.3 \mathrm{~Hz}\right)$, $115.5\left(\mathrm{~d}, J_{C-F}=22.7 \mathrm{~Hz}\right), 114.2\left(\mathrm{~d}, J_{C-F}=21.2 \mathrm{~Hz}\right), 109.9,71.8(\mathrm{~d}$, $\left.J_{C-F}=1.3 \mathrm{~Hz}\right)$, 70.14. HRMS: $\mathrm{m} / \mathrm{z}:[\mathrm{M}+\mathrm{H}]^{+}$calculated for $\mathrm{C}_{27} \mathrm{H}_{19} \mathrm{~F}_{2} \mathrm{NO}: 412.1507$, Found: 412.1509.

3ai. ${ }^{1} \mathrm{H} \mathrm{NMR}\left(400 \mathrm{MHz}, \mathrm{CDCl}_{3}\right) \delta 7.67(\mathrm{t}, J=1.8 \mathrm{~Hz}, 1 \mathrm{H}), 7.38$ (m, 1H), $7.33-7.28(\mathrm{~m}, 1 \mathrm{H}), 7.21-7.12(\mathrm{~m}, 2 \mathrm{H}), 7.08-6.95(\mathrm{~m}$, $7 \mathrm{H}), 6.92(\mathrm{t}, J=7.9 \mathrm{~Hz}, 1 \mathrm{H}), 6.82(\mathrm{~d}, J=7.8 \mathrm{~Hz}, 1 \mathrm{H}), 6.68(\mathrm{td}, J=$ $7.6,0.9 \mathrm{~Hz}, 1 \mathrm{H}), 6.63(\mathrm{t}, J=1.8 \mathrm{~Hz}, 1 \mathrm{H}), 6.53-6.47(\mathrm{~m}, 1 \mathrm{H}), 6.11$ (s, 1H), 4.32 (s, 1H). ${ }^{13} \mathrm{C}$ NMR $\left(100 \mathrm{MHz}, \mathrm{CDCl}_{3}\right) \delta 195.4,152.0$, $140.0,139.1,138.2,134.4,134.3,132.3,130.7,129.8,129.2$, 129.1, 128.8, 128.4, 128.0, 127.8, 127.6, 127.5, 127.4, 126.6, 124.9, 119.2, 110.0, 71.8, 70.2. HRMS: $m / z$ : $[\mathrm{M}+\mathrm{H}]^{+}$calculated for $\mathrm{C}_{27} \mathrm{H}_{19} \mathrm{Cl}_{2} \mathrm{NO}: 444.0916$, Found: 444.0915 .

3aj. ${ }^{1} \mathrm{H}$ NMR (400 MHz, $\left.\mathrm{CDCl}_{3}\right) \delta 7.85(\mathrm{t}, J=1.7 \mathrm{~Hz}, 1 \mathrm{H}), 7.53$ (m, 1H), $7.33(\mathrm{~d}, J=8.0 \mathrm{~Hz}, 1 \mathrm{H}), 7.24-7.16(\mathrm{~m}, 2 \mathrm{H}), 7.11-7.00$ $(\mathrm{m}, 4 \mathrm{H}), 7.00-6.93(\mathrm{~m}, 3 \mathrm{H}), 6.89-6.80(\mathrm{~m}, 2 \mathrm{H}), 6.76(\mathrm{t}, J=1.7$ $\mathrm{Hz}, 1 \mathrm{H}), 6.69(\mathrm{~m}, 1 \mathrm{H}), 6.55$ (d, J = 7.9 Hz, 1H), $6.11(\mathrm{~s}, 1 \mathrm{H}), 4.27$ (s, 1H). ${ }^{13} \mathrm{C}$ NMR $\left(100 \mathrm{MHz}, \mathrm{CDCl}_{3}\right) \delta 195.2,152.0,140.3,139.0$, 138.3, 135.2, 133.7, 131.3, 130.4, 129.8, 129.5, 129.3, 129.2, 128.0, 127.8, 127.7, 127.5, 127.0, 124.8, 122.5, 122.5, 119.2, 110.0, 71.8, 70.2. HRMS: $m / z:[\mathrm{M}+\mathrm{H}]^{+}$calculated for $\mathrm{C}_{27} \mathrm{H}_{20} \mathrm{Br}_{2} \mathrm{NO}: 531.9906$, Found: 531.9902.

3ak. ${ }^{1} \mathrm{H}$ NMR (400 MHz, $\left.\mathrm{CDCl}_{3}\right) \delta 7.33-7.19(\mathrm{~m}, 5 \mathrm{H}), 7.15(\mathrm{t}$, $J=7.6 \mathrm{~Hz}, 1 \mathrm{H}), 7.03(\mathrm{~d}, J=7.4 \mathrm{~Hz}, 1 \mathrm{H}), 6.78(\mathrm{q}, J=6.6 \mathrm{~Hz}, 2 \mathrm{H})$, $4.90(\mathrm{~s}, 1 \mathrm{H}), 4.13(\mathrm{~s}, 1 \mathrm{H}), 2.17-2.03(\mathrm{~m}, 2 \mathrm{H}), 1.98(\mathrm{t}, J=7.4 \mathrm{~Hz}$, $2 \mathrm{H}), 1.53-1.38(\mathrm{~m}, 1 \mathrm{H}), 1.20-1.08(\mathrm{~m}, 1 \mathrm{H}), 1.07-0.97(\mathrm{~m}$, $1 \mathrm{H}), 0.94(\mathrm{t}, J=7.2 \mathrm{~Hz}, 3 \mathrm{H}), 0.89-0.78(\mathrm{~m}, 1 \mathrm{H}), 0.50(\mathrm{t}, J=7.4$ $\mathrm{Hz}, 3 \mathrm{H}) .{ }^{13} \mathrm{C}$ NMR $\left(100 \mathrm{MHz}, \mathrm{CDCl}_{3}\right) \delta 210.5,151.4,140.3,129.6$, $128.5,128.46,127.9,126.9,125.8,118.9,109.4,70.8,67.5,43.7$, 38.6, 18.2, 16.7, 14.6, 13.5. HRMS: $m / z:[\mathrm{M}+\mathrm{H}]^{+}$calculated for $\mathrm{C}_{21} \mathrm{H}_{26} \mathrm{NO}$ : 308.2009, Found: 308.2009.

4 was prepared according to the general procedure but additional $\mathrm{Zn}(\mathrm{OTf})_{2}(20 \mathrm{~mol} \%)$ was used. ${ }^{1} \mathrm{H}$ NMR $(400 \mathrm{MHz}$, Acetone) $\delta 10.70(\mathrm{~s}, 1 \mathrm{H}), 7.64(\mathrm{~d}, J=8.0 \mathrm{~Hz}, 1 \mathrm{H}), 7.56(\mathrm{t}, J=8.2 \mathrm{~Hz}$, 3H), $7.50-7.44(\mathrm{~m}, 4 \mathrm{H}), 7.43-7.33(\mathrm{~m}, 4 \mathrm{H}), 7.25$ (t, J=7.6 Hz, $1 \mathrm{H}), 7.14(\mathrm{t}, J=7.5 \mathrm{~Hz}, 1 \mathrm{H})$. The NMR data agree with those in a literature report [50].

\section{Results and discussion}

We initiated our investigation with optimization studies on the coupling between $N$-phenyl nitrone 1a and diphenylacetylene (2a) in the presence of $\left[\mathrm{RhCp}^{*} \mathrm{Cl}_{2}\right]_{2}$ catalyst (4 mol\%, Table 1). The reaction proceeded in poor yield and low selectivity in DCE in the absence of any acid additive (entry 1). Addition of PivOH or $\mathrm{AcOH}$ increased the conversion and the selectivity (entries 2 and 3), and the major product was identified as a 2,3,3-trisubstituted indoline (3aa). Product 3aa was fully characterized, including by X-ray crystallography (not shown). However, diastereomer 3aa' and indole 4 were also detected as the minor products, but no indenone product has been observed. These products were generated via exo $\mathrm{C}-\mathrm{H}$ activation assisted by the $\mathrm{O}$-coordination. It should be noted that the conditions that were optimal for related $\mathrm{C}-\mathrm{H}$ activation-O-atom transfer reaction between quinoline $N$-oxide and an alkyne only gave poor results (entries 2,3) [47]. Switching to other Ag salts or to $\mathrm{Zn}(\mathrm{OTf})_{2}$ only gave negative results (entry 4). Significant solvent effects have been observed. Using DCM as the solvent, both the selectivity and the conversion of the reaction were improved, and product 3aa was isolated in 63\% yield. Extensive screening of solvents revealed that the yield was optimal in ethyl acetate (68\%, entry 8). Control experiments also proved that the Rh catalyst was necessary; omission of the $\mathrm{Rh}$ catalyst or switching to the Ir congener only resulted in decomposition of the starting nitrone.

With the optimized conditions established, we next explored the scope and generality of this coupling (Table 2). The scope of the nitrone was first examined in the coupling with diphenylacetylene. Introduction of electron-donating, -withdrawing, and halogen groups at the para position of the $\mathrm{N}$-phenyl ring is fully tolerated. In all cases, both diastereomeric products were generated, and diastereomeric ratio of the crude products has been determined by ${ }^{1} \mathrm{H}$ NMR spectroscopy. The major product corresponds to that with the two phenyl groups in a cis orientation, and in most cases the major product

\section{Table 1}

Optimization studies.

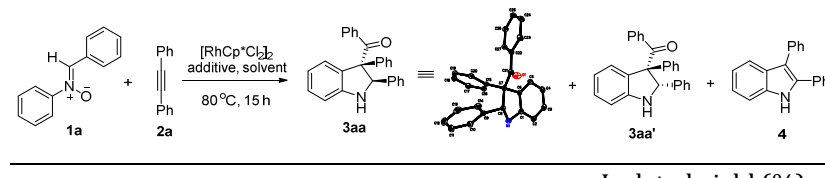

\begin{tabular}{|c|c|c|c|c|c|c|}
\hline \multirow{2}{*}{ Entry } & \multirow{2}{*}{ Additive } & \multirow{2}{*}{ Acid } & \multirow{2}{*}{ Solvent } & \multicolumn{3}{|c|}{ Isolated yield (\%) } \\
\hline & & & & 3aa & 3aa' & 4 \\
\hline 1 & $\mathrm{AgSbF}_{6}$ & - & DCE & $<5$ & 12 & $<5$ \\
\hline 2 & $\mathrm{AgSbF}_{6}$ & PivOH & DCE & 36 & $<5$ & $<5$ \\
\hline 3 & $\mathrm{AgSbF}_{6}$ & $\mathrm{AcOH}$ & DCE & 24 & $<5$ & $<5$ \\
\hline 4 & $\mathrm{Zn}(\mathrm{OTf})_{2}$ & PivOH & DCE & trace & trace & $<5$ \\
\hline $5^{a}$ & $\operatorname{AgNTf}_{2}$ & PivOH & DCE & nd & nd & trace \\
\hline 6 & $\mathrm{AgSbF}_{6}$ & PivOH & DCM & 63 & 9 & $<5$ \\
\hline 7 & $\mathrm{AgSbF}_{6}$ & $\mathrm{PivOH}$ & dioxane & 49 & 8 & $<5$ \\
\hline 8 & $\mathrm{AgSbF}_{6}$ & PivOH & EtOAc & 68 & 9 & $<5$ \\
\hline 9 & $\mathrm{AgSbF}_{6}$ & PivOH & Acetone & 40 & 7 & $<5$ \\
\hline 10 & $\mathrm{AgSbF}_{6}$ & $\mathrm{PivOH}$ & $t-\mathrm{AmOH}$ & trace & trace & $<5$ \\
\hline $11^{b}$ & $\mathrm{AgSbF}_{6}$ & $\mathrm{PivOH}$ & DCE & nd & nd & nd \\
\hline $12^{\mathrm{c}}$ & $\mathrm{AgSbF}_{6} / \mathrm{Zn}(\mathrm{OTf})_{2}$ & $\mathrm{PivOH}$ & DCE & trace & trace & 40 \\
\hline
\end{tabular}

Reaction conditions: nitrone 1a $(0.2 \mathrm{mmol})$, diphenylacetylene $(0.3$ mmol), $\left[\mathrm{Cp}^{*} \mathrm{RhCl}_{2}\right]_{2}$ (4 mol\%), additive (16 mol\%), acid (2 equiv) in solvent $(2 \mathrm{~mL}), 80{ }^{\circ} \mathrm{C}$ for $15 \mathrm{~h}$. a $\left[\mathrm{IrCp}^{*} \mathrm{Cl}_{2}\right]_{2}$ was used as a catalyst. ${ }^{\mathrm{b}} \mathrm{No}$ catalyst was used. ${ }^{\mathrm{c}}$ Additional $\mathrm{Zn}(\mathrm{OTf})_{2}(20 \mathrm{~mol} \%)$ was used. 
Table 2

Scope of substrates.

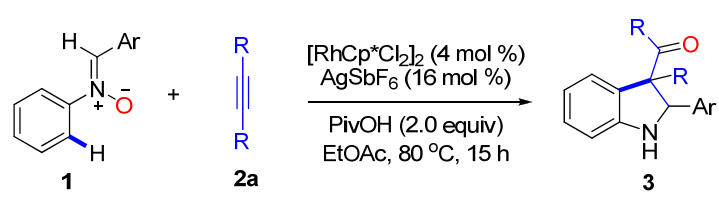

\begin{tabular}{|c|c|c|c|c|c|c|c|c|c|}
\hline Entry & Product & & Isolated yield (\%) & $\mathrm{dr}^{\mathrm{a}}$ & Entry & Product & & Isolated yield (\%) & $\mathrm{dr}^{\mathrm{a}}$ \\
\hline 1 & & 3аa & 68 & 7.1:1 & 13 & & $3 \mathrm{ma}$ & 81 & $5.5: 1$ \\
\hline 2 & & $3 \mathbf{b a}$ & 54 & $5.9: 1$ & 14 & & 3na & 50 & $5.2: 1$ \\
\hline 3 & & $3 \mathbf{c a}$ & 61 & $4.2: 1$ & 15 & & 3ab & 76 & 7.0:1 \\
\hline 4 & & 3da & 69 & $4.0: 1$ & 16 & & 3ac & 50 & 8.3:1 \\
\hline 5 & & 3ea & 73 & $3.7: 1$ & 17 & & 3ad & 75 & 7.9:1 \\
\hline 6 & & $3 f a$ & 79 & $6.0: 1$ & 18 & & 3ae & 66 & $6.6: 1$ \\
\hline 7 & & 3ga & 45 & 7.9:1 & 19 & & 3af & 72 & $6.0: 1$ \\
\hline 8 & & 3ha & 64 & $9.9: 1$ & 20 & & 3ag & 61 & $7.2: 1$ \\
\hline 9 & & $3 \mathbf{i a}$ & 58 & 7.1:1 & 21 & & 3ah & 53 & $5.9: 1$ \\
\hline 10 & & $3 \mathbf{j a}$ & 61 & $6.2: 1$ & 22 & & 3ai & 48 & $5.9: 1$ \\
\hline 11 & & 3ka & 71 & $6.2: 1$ & 23 & & 3aj & 56 & $5.5: 1$ \\
\hline 12 & & 3la & 76 & $8.2: 1$ & 24 & & $3 \mathbf{a k}^{\mathrm{b}}$ & 56 & $5.5: 1$ \\
\hline
\end{tabular}

Reaction conditions: nitrone (0.2 mmol), alkyne (0.3 mmol), [Cp* $\left.\mathrm{RhCl}_{2}\right]_{2}(4 \mathrm{~mol} \%), \mathrm{AgSbF}_{6}(16 \mathrm{~mol} \%)$, PivOH $\left(2.0\right.$ equiv), ethyl acetate $(2 \mathrm{~mL}), 80{ }^{\circ} \mathrm{C}$, $15 \mathrm{~h}$, sealed tube under $\mathrm{N}_{2 .}{ }^{\text {a }}$ trans:cis ratio. ${ }^{\mathrm{b}}$ DCM solvent was used in a sealed tube. 
can be isolated by column chromatography, but the minor isomer overlaps with the major one or other impurities and defies further purification. Introduction of meta substituents such as methyl, tert-Bu and $\mathrm{Ph}$ resulted in steric bias and the $\mathrm{C}-\mathrm{H}$ bond at the less hindered position underwent coupling with high selectivity. In contrast, variation of the spectating C-phenyl ring in the nitrone is also tolerated. Introduction of electron-donating and halogen group or using a heteroaryl group caused no negative effect. In all cases, the reaction gave nearly full conversion and $d r$ ranges from 3.7-9.9:1, and the major product was isolated in moderate to high yield.

The scope of the alkyne substrate was next explored. Diphenylacetylenes bearing substituents such as $m$ - and $p$-halogen, -OMe, and -alkyl groups all reacted with $\mathbf{1 a}$ in moderate to good yield and the $d r$ ranges from 5.5-8.3:1. The alkyne is not limited to diaryl substituents, and 4-octynes reacted under the optimized conditions (in DCM) with moderate yield. It should be noted that aryl- and alkyl-mixed alkynes such as $\mathrm{PhC} \equiv \mathrm{CMe}$ are also viable; however, the complication of regio- and stereoselectivity rendered it difficult to isolate the desired product.

To probe the intramolecularity of the 0-atom transfer process, the coupling of $\mathbf{1 a}$ and 4-octyne were performed in the presence of $\mathrm{H}_{2} \mathrm{O}^{18}$ (2 equiv). MS analysis of the product indicated that no $\mathrm{O}^{18}$ atom was incorporated, and this observation is in line with that in our previously reported coupling between quinoline $N$-oxide and alkynes, where the $\mathrm{O}$-atom transfer is intramolecular. To probe the $\mathrm{C}-\mathrm{H}$ activation process, kinetic isotope effect has been measured from the competitive coupling between $\mathbf{1 a}$ and $\mathbf{1 a}-d_{5}$ in equimolar ratio (Eq. 1). The reaction was performed at $40^{\circ} \mathrm{C}$ in DCM and was stopped after with low conversion (ca. 15\%). The crude product was purified by chromatography to afford a mixture of 3ak and 3ak- $d_{4} .{ }^{1} \mathrm{H}$ NMR analysis of the mixture revealed a KIE value of 5.3, indicating that $\mathrm{C}-\mathrm{H}$ cleave is likely involved in the rate-limiting step.

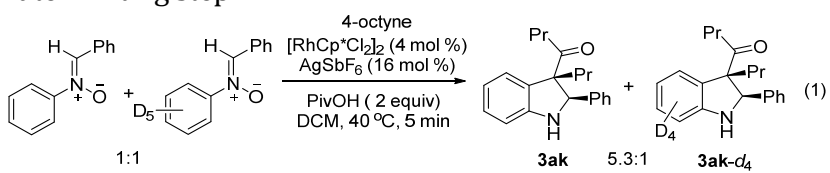

On the basis of previous reports, a plausible catalytic cycle is proposed (Scheme 1) starting from an active $\mathrm{RhCp}^{*} \mathrm{X}_{2}\left(\mathrm{X}=\mathrm{SbF}_{6}\right.$ or OPiv) catalyst. O-coordination-assisted $\mathrm{C}-\mathrm{H}$ activation of the nitrone affords a rhodacyle $\mathbf{A}$. Coordination of the alkyne followed by migratory insertion of the $\mathrm{Rh}-\mathrm{C}$ bond generates a seven-membered rhodacyclic intermediate $\mathbf{B}$, which is proposed to undergo $\mathrm{C}-\mathrm{O}$ reductive elimination to give a cationic six-membered heterocycle (C) [47] together with a $\mathrm{Cp} * \mathrm{Rh}(\mathrm{I})$ species. The $\mathrm{N}-\mathrm{O}$ bond in heterocycle $\mathbf{C}$ then oxidatively adds back to the $\mathrm{Rh}(\mathrm{I})$ to generated a $\mathrm{Rh}(\mathrm{III})$ enolate species $\mathbf{E}$, which accounts for the oxygen transfer. Protonolysis of the Rh-O bond in $\mathbf{E}$ by an acid (HX) releases an imine $\mathbf{F}$. The fate of $\mathbf{F}$ can be found in two subsequent pathways. When sufficient water and a Lewis acid $\left(\mathrm{Cu}(\mathrm{OAc})_{2}\right.$ and $\mathrm{Zn}(\mathrm{OTf})_{2}$ ) are present, hydrolysis is followed to produce an aniline with a pendent ketone moiety, intermolecular condensation of which furnishes the indole byproduct.
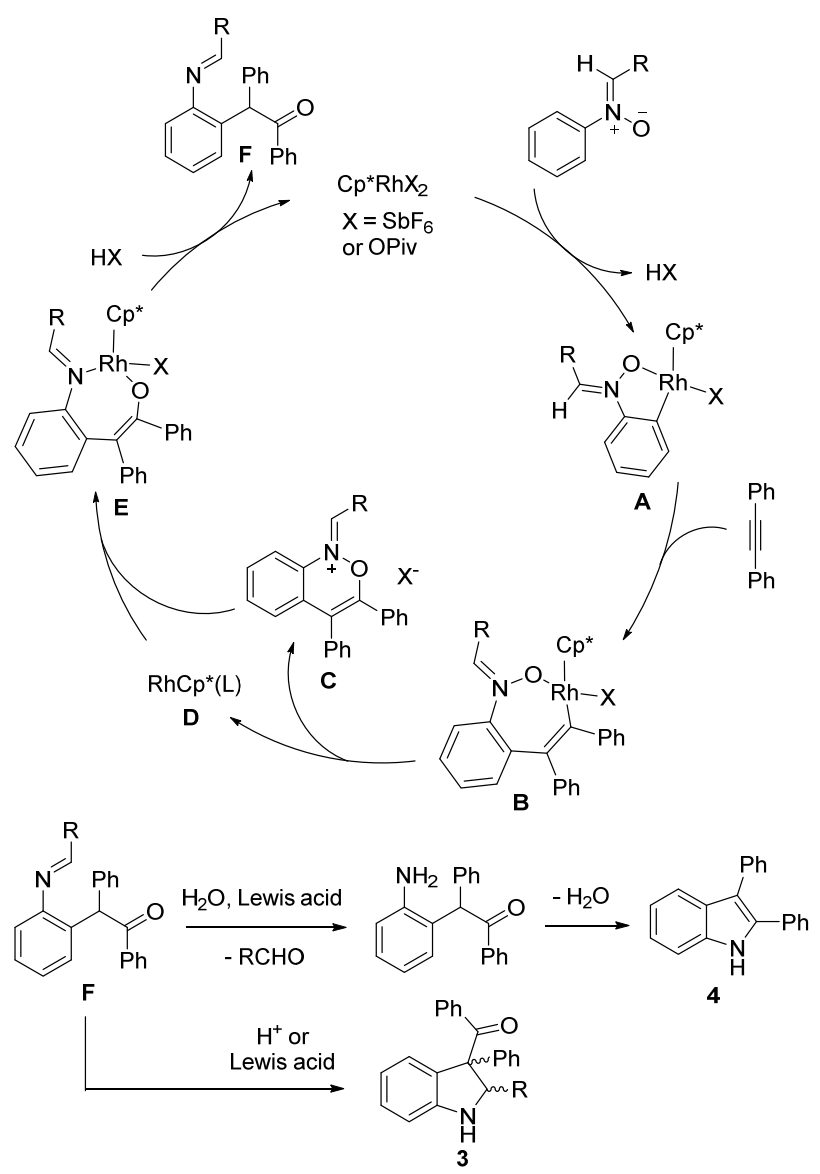

Scheme 1. Proposed mechanism of Rh(III)-catalyzed coupling of nitrones with alkynes.

Alternatively, intramolecular nucleophilic attack of the enol form of the ketone yields the final indoline product as a mixture of diastereomers.

\section{Conclusions}

We have achieved the $\mathrm{Rh}(\mathrm{III})$-catalyzed $\mathrm{C}-\mathrm{H}$ activation of $\mathrm{N}$-aryl nitrones in the coupling with alkynes with rare oxygen atom transfer under redox-neutral conditions. The reaction proceeded under relatively mild conditions with high chemoselectivity and moderate to good diastereoselectivity and provided trisubstituted protic indolines as synthetically useful products. In all cases, the $\mathrm{C}-\mathrm{H}$ activation occurred at the $\mathrm{N}$-aryl ring (exo $\mathrm{C}-\mathrm{H}$ activation), which differs from the endo selectivity in our previously reported $\mathrm{Rh}(\mathrm{III})$-catalyzed $\mathrm{C}-\mathrm{H}$ activation of nitrones. The chemoselectivity also stays contrast to that previously reported in Rh(III) catalysis. A broad scope of substrates has been established, and this method may find applications in the synthesis of complex structures.

\section{References}

[1] Chen X, Engle K M, Wang D H, Yu J Q. Angew Chem Int Ed, 2009, 48: 5094

[2] Yeung C S, Dong V M. Chem Rev, 2011, 111: 1215

[3] Wencel-Delord J, Droege T, Liu F, Glorius F. Chem Soc Rev, 2011, 


\section{Graphical Abstract}

Chin. J. Catal., 2015, 36: 925-932 doi: 10.1016/S1872-2067(15)60866-X

Rh(III)-catalyzed coupling of nitrones with alkynes for the synthesis of indolines

Lingheng Kong, Fang Xie, Songjie Yu, Zisong Qi, Xingwei Li*

Dalian Institute of Chemical Physics, Chinese Academy of Sciences

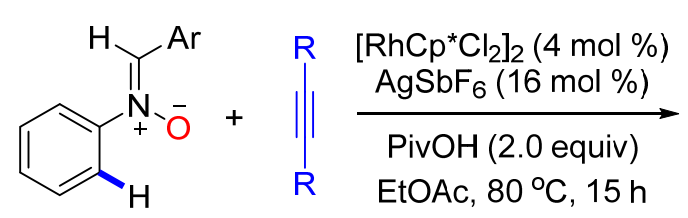<smiles>[R]C(=O)C1([R])c2ccccc2NC1[Te]</smiles>

25 examples
-Combination of $\mathrm{C}-\mathrm{H}$ activation with OAT -High chemoselectivity -Moderate to good dr

Rh-catalyzed redox-neutral coupling between $N$-aryl nitrones and alkynes has been achieved under relatively mild conditions. The reaction proceeded via $\mathrm{C}$ - $\mathrm{H}$ activation at the $\mathrm{N}$-aryl ring with subsequent $\mathrm{O}$-atom transfer, affording trisubstituted indolines in good chemoselectivity and moderate to good diasteroselectivity.

40: 4740

[4] Sun C L, Li B J, Shi Z J. Chem Rev, 2011, 111: 1293

[5] Yamaguchi J, Yamaguchi A D, Itami K. Angew Chem Int Ed, 2012, 51: 8960

[6] McMurray L, O'Hara F, Gaunt M J. Chem Soc Rev, 2011, 40: 1885

[7] Cho S H, Kim J Y, Kwak J, Chang S. Chem Soc Rev, 2011, 40: 5068

[8] Ackermann L. Acc Chem Res, 2014, 47: 281

[9] Ackermann L. Chem Rev, 2011, 111: 1315

[10] Xie F, Qi Z S, Yu S J, Li X W.J Am Chem Soc, 2014, 136: 4780

[11] Kim J Y, Park S H, Ryu J, Cho S H, Kim S H, Chang S. J Am Chem Soc, 2012, 134: 9110

[12] Zhen W C, Du Z Y, Li X W. Chin J Catal (甄文萃, 杜正银, 李兴伟. 催 化学报), 2013, 34: 679

[13] Zhao D, Nimphius C, Lindale M, Glorius F. Org Lett, 2013, 15: 4504

[14] Qi Z S, Li X W. Chin J Catal (戚自松, 李兴伟. 催化学报), 2015, 36: 48

[15] Zhang F, Spring D R. Chem Soc Rev, 2014, 43: 6906

[16] Kuhl N, Schröder N, Glorius F. Adv Synth Catal, 2014, 356: 1443

[17] Colby D A, Tsai A S, Bergman R G, Ellman J A. Acc Chem Res, 2012, 45: 814

[18] Song G Y, Wang F, Li X W. Chem Soc Rev, 2012, 41: 3651

[19] Colby D A, Bergman R G, Ellman J A. Chem Rev, 2010, 110: 624

[20] Guimond N, Gouliaras C, Fagnou K. J Am Chem Soc, 2010, 132: 6908

[21] Guimond N, Gorelsky S I, Fagnou K. J Am Chem Soc, 2011, 133: 6449

[22] Patureau F W, Glorius F. Angew Chem Int Ed, 2011, 50: 1977

[23] Rakshit S, Grohmann C, Besset T, Glorius F. J Am Chem Soc, 2011, 133: 2350

[24] Huang H W, Ji X C, Wu W Q Jiang H F. Chem Soc Rev, 2015, 44: 1155

[25] Hyster T K, Rovis T. Chem Commun, 2011, 47: 11846

[26] Martin R M, Bergman R G, Ellman J A. J Org Chem, 2012, 77: 2501

[27] Wang H G, Glorius F. Angew Chem Int Ed, 2012, 51: 7318

[28] Neely J M, Rovis T. J Am Chem Soc, 2013, 135: 66

[29] Huckins J R, Bercot E A, Thiel O R, Hwang T L, Bio M M. J Am Chem Soc, 2013, 135: 14492

[30] Zhang X P, Chen D, Zhao M, Zhao J, Jia A Q, Li X W. Adv Synth Catal, 2011, 353: 719
[31] Huang X L, Huang J S, Du C L, Zhang X Y, Song F J, You J S. Angew Chem Int Ed, 2013, 52: 12970

[32] Cui S L, Zhang Y, Wu Q F. Chem Sci, 2013, 4: 3421

[33] Liu G X, Shen Y Y, Zhou Z, Lu X Y. Angew Chem Int Ed, 2013, 52: 6033

[34] Shen Y Y, Liu G X, Zhou Z, Lu X Y. Org Lett, 2013, 15: 3366

[35] Shi J J, Zhao G G, Wang X W, Xu H E, Yi W. Org Biomol Chem, 2014, 12: 6831

[36] Chen Y, Wang D Q, Duan P P, Ben R, Dai L, Shao X R, Hong M, Zhao J, Huang Y. Nat Commun, 2014, 5: 4610

[37] Chuang S C, Gandeepan P, Cheng C H. Org Lett, 2013, 15: 5750

[38] Liu B Q, Song C, Sun C, Zhou S G, Zhu J. J Am Chem Soc, 2013, 135: 16625

[39] Zhao D B, Shi Z Z, Glorius F. Angew Chem Int Ed, 2013, 52: 12426

[40] Zheng L Y, Hua R M, Chemistry, 2014, 20: 2352

[41] Wang C M, Sun H, Fang Y, Huang Y. Angew Chem Int Ed, 2013, 52: 5795

[42] Matsuda T, Tomaru Y. Tetrahedron Lett, 2014, 55: 3302

[43] Huang X C, Yang X H, Song R J, Li J H. J Org Chem, 2014, 79: 1025

[44] Fan Z L, Song S S, Li W, Geng K J, Xu Y J, Miao Z H, Zhang A. Org Lett, 2015, 17: 310

[45] Zhang Q R, Huang J R, Zhang W, Dong L. Org Lett, 2014, 16: 1684

[46] Yu S J, Liu S, Lan Y, Wan B S, Li X W. J Am Chem Soc, 2015, 137: 1623

[47] Zhang XY, Qi Z S, Li X W. Angew Chem Int Ed, 2014, 53: 10794

[48] Sharma U, Park Y, Chang S. J Org Chem, 2014, 79: 9899

[49] Qi Z S, Wang M, Li X W. Org Lett, 2013, 15: 5440

[50] Wang C X, Wang D P, Yan H, Wang H L, Pan B, Xin X Y, Li X C, Wu F, Wan B S. Angew Chem Int Ed, 2014, 53: 11940

[51] Ren Z, Mo F Y, Dong G B. J Am Chem Soc, 2012, 134: 16991

[52] Mawo R Y, Mustakim S, Young V G, Hoffmann M R, Smoliakova I P. Organometallics, 2007, 26: 1801

[53] Bielsa R, Navarro R, Urriolabeitia E P, Lledós A. Inorg Chem, 2007, 46: 10133

[54] Williams R M, Cox R J. Acc Chem Res, 2003, 36: 127

[55] Zhang D, Song H, Qin Y. Acc Chem Res, 2011, 44: 447

[56] Takano S, Ogasawara K. Alkaloids, 1989, 36: 225

[57] Mio M J, Kopel L C, Braun J B, Gadzikwa T L, Hull K L, Brisbois R G, Markworth C J, Grieco P A. Org Lett, 2002, 4: 3199

[58] Dateer R B, Chang S. J Am Chem Soc, 2015, 137: 4908 


\section{三价铑催化硝酮与炔的碳氢活化偶联合成二氢吲哚}

孔令恒, 谢芳, 于松杰, 戚自松, 李兴伟 ${ }^{*}$

中国科学院大连化学物理研究所, 辽宁大连 116023

摘要: 三价铑在氧化还原中性条件下催化硝酮与炔发生偶联, 经过氮芳环的碳氢键活化和氧转移可以高化学选择性、中等到良好 非对映选择性的得到三取代二氢吲哚.

关键词: 三价铑；碳氢活化；氧转移；氮芳基硝酮

收稿日期: 2015-04-22. 接受日期: 2015-04-30. 出版日期: 2015-07-20.

*通讯联系人. 电话: (0411)84379089; 电子信箱: xwli@dicp.ac.cn

基金来源：中国科学院大连化学物理研究所; 国家自然科学基金(21272231, 21472186, 21372266).

本文的英文电子版由Elsevier出版社在ScienceDirect上出版(http://www.sciencedirect.com/science/journal/18722067). 